\title{
SOME IDENTITIES FOR A SEQUENCE OF UNNAMED POLYNOMIALS CONNECTED WITH THE BELL POLYNOMIALS
}

\author{
FENG QI
}

Institute of Mathematics, Henan Polytechnic University, Jiaozuo City, Henan Province, 454010, China; College of Mathematics, Inner Mongolia University for Nationalities, Tongliao City, Inner Mongolia Autonomous Region, 028043, China; Department of Mathematics, College of Science, Tianjin Polytechnic University, Tianjin City, 300387, China

\section{DA-WEI NIU}

Department of Mathematics, East China Normal University, Shanghai City, 200241, China

\section{BAI-NI GUO}

School of Mathematics and Informatics, Henan Polytechnic University, Jiaozuo City, Henan Province, 454010, China

\begin{abstract}
In the paper, using two inversion theorems for the Stirling numbers and binomial coefficients, employing properties of the Bell polynomials of the second kind, and utilizing a higher order derivative formula for the ratio of two differentiable functions, the authors present two explicit formulas, a determinantal expression, and a recursive relation for a sequence of unnamed polynomials, derive two identities connecting the sequence of unnamed polynomials with the Bell polynomials, and recover a known identity connecting the sequence of unnamed polynomials with the Bell polynomials.
\end{abstract}

E-mail addresses: qifeng618@gmail.com, qifeng618@hotmail.com, qifeng618@qq.com, nnddww@gmail.com, bai.ni.guo@gmail.com, bai.ni.guo@hotmail.com

2010 Mathematics Subject Classification. Primary 11B83; Secondary 05A15, 11A25, 11B65, 11B73, 11C08, 33B10.

Key words and phrases. identity; Bell polynomial; unnamed polynomial; explicit formula; inversion theorem; Stirling number; binomial coefficient.

This paper was typeset using $\mathcal{A M}_{\mathcal{M}}$-LATEX. 


\section{Motivations}

In [4, pp. 257-258] and [5], the functions in

$$
F(t, x)=\frac{1}{\sqrt{1-t}} \exp \left[x\left(\frac{1}{\sqrt{1-t}}-1\right)\right]=\sum_{n=0}^{\infty} h_{n}(x) \frac{t^{n}}{n !}
$$

were considered. In [4, pp. 257-258], it was pointed out that the unnamed polynomials $h_{n}(x)$ satisfy

$$
h_{n}(x)=\frac{1}{n !} \frac{x}{e^{x}}\left[\frac{\mathrm{d}}{\mathrm{d}\left(x^{2}\right)}\right]^{n}\left(x^{2 n-1} e^{x}\right) .
$$

In [5], it was pointed out that the unnamed polynomials $h_{n}(x)$ and the Bell polynomials $B_{n}(x)$ are connected by the identity

$$
\sum_{k=0}^{n}\left(\begin{array}{l}
n \\
k
\end{array}\right) B_{k}(x)=(-2)^{n} \sum_{k=0}^{n}(-1)^{k} h_{k}(x) S(n, k), \quad n \geq 0,
$$

where the Bell polynomials $B_{k}(x)$ can be generated by

$$
e^{x\left(e^{t}-1\right)}=\sum_{k=0}^{\infty} B_{k}(x) \frac{t^{k}}{k !}
$$

and the Stirling numbers of the second kind $S(n, k)$ can be generated by

$$
\frac{\left(e^{x}-1\right)^{k}}{k !}=\sum_{n=k}^{\infty} S(n, k) \frac{x^{n}}{n !}, \quad k \in\{0\} \cup \mathbb{N} .
$$

It was pointed out in [4. pp. 257-258] that the expression 1.2 had been applied in 1937 to the theory of hyperbolic differential equations. It was also pointed out in [10] that there have been some studies on interesting applications of Bell polynomials $B_{k}(x)$ in soliton theory, including links with bilinear and trilinear forms of nonlinear differential equations which possess soliton solutions. See, for example, 6, 7, 8. Therefore, applications of the Bell polynomials $B_{k}(x)$ to integrable nonlinear equations are greatly expected and any amendment on multi-linear forms of soliton equations, even on exact solutions, would be beneficial to interested audiences in the research community.

To simplify main results in [5], among other things, the following conclusions were established in the newly published paper [10].

Theorem 1.1 ([10, Theorem 1]). For $n \geq 0$, the nth derivative of the generating function $F(t, x)$ of the polynomials $h_{n}(x)$ in (1.1) can be computed by

$$
\frac{\mathrm{d}^{n} F(t, x)}{\mathrm{d} t^{n}}=\frac{F(t, x)}{2^{n}} \sum_{k=0}^{n}\left[\sum_{\ell=k}^{n} \ell !(2 n-2 \ell-1) ! !\left(\begin{array}{c}
2 n-\ell-1 \\
2(n-\ell)
\end{array}\right)\left(\begin{array}{l}
\ell \\
k
\end{array}\right)\right] \frac{x^{k}}{k !(1-t)^{n+k / 2}},
$$

where the double factorial of negative odd integers $-(2 n+1)$ is defined by

$$
(-2 n-1) ! !=\frac{(-1)^{n}}{(2 n-1) ! !}=(-1)^{n} \frac{2^{n} n !}{(2 n) !}, \quad n \geq 0
$$

and the conventions $\left(\begin{array}{l}0 \\ 0\end{array}\right)=1$ and $\left(\begin{array}{l}p \\ q\end{array}\right)=0$ for $q>p \geq 0$ are adopted. Consequently, the polynomials $h_{n}(x)$ for $n \geq 0$ can be expressed as

$$
h_{n}(x)=\frac{1}{2^{n}} \sum_{k=0}^{n}\left[\sum_{\ell=k}^{n} \ell !(2 n-2 \ell-1) ! !\left(\begin{array}{c}
2 n-\ell-1 \\
2(n-\ell)
\end{array}\right)\left(\begin{array}{l}
\ell \\
k
\end{array}\right)\right] \frac{x^{k}}{k !} .
$$


Theorem 1.2 ([10, Theorem 3]). For $n \geq 0$, the Bell polynomials $B_{n}(x)$ and the polynomials $h_{n}(x)$ can be expressed each other by

$$
B_{n}(x)=(-1)^{n} \sum_{\ell=0}^{n}(-1)^{\ell}\left[\sum_{k=\ell}^{n} 2^{k}\left(\begin{array}{l}
n \\
k
\end{array}\right) S(k, \ell)\right] h_{\ell}(x)
$$

and

$$
h_{n}(x)=\frac{1}{2^{n}} \sum_{\ell=0}^{n}\left[\sum_{k=\ell}^{n}\left(\begin{array}{l}
n \\
k
\end{array}\right)(2 n-2 k-1) ! ! 2^{k-\ell} s(k, \ell)\right] B_{\ell}(x),
$$

where $s(n, k)$ for $n \geq k \geq 0$, which can be generated by

$$
\frac{[\ln (1+x)]^{k}}{k !}=\sum_{n=k}^{\infty} s(n, k) \frac{x^{n}}{n !}, \quad|x|<1,
$$

stand for the Stirling numbers of the first kind.

Theorem 1.3 ([10, Theorem 4]). For $n \geq 0$, the Bell polynomials $B_{n}(x)$ and the polynomials $h_{n}(x)$ satisfy the identities

$$
\sum_{k=0}^{n}\left(\begin{array}{l}
n \\
k
\end{array}\right) 2^{k}(2 n-2 k-3) ! ! h_{k}(x)=-2^{n} \sum_{k=0}^{n} \frac{s(n, k)}{2^{k}} B_{k}(x)
$$

and

$$
\sum_{k=0}^{n}\left(\begin{array}{l}
n \\
k
\end{array}\right)\left[\sum_{\ell=0}^{n-k} \frac{s(n-k, \ell)}{2^{\ell}} B_{\ell}(-x)\right] h_{k}(x)=\frac{(2 n-1) ! !}{2^{n}} .
$$

In this paper, using two inversion theorems for the Stirling numbers $s(n, k)$ and $S(n, k)$ and binomial coefficients $\left(\begin{array}{l}n \\ k\end{array}\right)$, employing properties of the Bell polynomials of the second kind $\mathrm{B}(n, k)$, and utilizing a higher order derivative formula for the ratio of two differentiable functions, the authors present two explicit formulas, a determinantal expression, and a recursive relation for $h_{n}(x)$, derive two identities connecting $h_{n}(x)$ with $B_{n}(x)$, and recover the identity (1.3).

Our main results can be stated as the following four theorems.

Theorem 1.4. For $n \geq 0$, the polynomials $h_{n}(x)$ satisfy

$$
h_{n}(x)=\frac{1}{n !} \sum_{k=0}^{n} \frac{(-1)^{k}}{2^{k}}\left(\begin{array}{l}
n \\
k
\end{array}\right)\left\langle n-\frac{1}{2}\right\rangle_{n-k} \sum_{\ell=0}^{k}(-1)^{\ell}[2(k-\ell)-1] ! !\left(\begin{array}{c}
2 k-\ell-1 \\
2(k-\ell)
\end{array}\right) x^{\ell},
$$

where

is the falling factorial.

$$
\langle x\rangle_{n}=\prod_{k=0}^{n-1}(x-k)= \begin{cases}x(x-1) \cdots(x-n+1), & n \geq 1 \\ 1, & n=0\end{cases}
$$

Theorem 1.5. For $n \geq 0$, the Bell polynomials $B_{n}(x)$ and $h_{n}(x)$ satisfy

$$
B_{n}(x)=-2^{n} \sum_{k=0}^{n} \frac{S(n, k)}{2^{k}} \sum_{\ell=0}^{k}\left(\begin{array}{l}
k \\
\ell
\end{array}\right) 2^{\ell}(2 k-2 \ell-3) ! ! h_{\ell}(x) .
$$

Theorem 1.6. For $n \geq 0$, the identity 1.3 is valid and

$$
h_{n}(x)=\sum_{k=0}^{n}(-1)^{n-k} \frac{s(n, k)}{2^{k}} \sum_{\ell=0}^{k}\left(\begin{array}{l}
k \\
\ell
\end{array}\right) B_{\ell}(x) .
$$


Theorem 1.7. For $n \geq 0$, the polynomials $h_{n}(x)$ can be computed by the determinantal expression

$$
\left.h_{n}(x)=(-1)^{n} \mid \begin{array}{cccccc}
1 & \lambda_{0}(x) & 0 & \cdots & 0 & 0 \\
\frac{1 ! !}{2} & \lambda_{1}(x) & \lambda_{0}(x) & \cdots & 0 & 0 \\
\frac{3 ! !}{2^{2}} & \lambda_{2}(x) & \left(\begin{array}{l}
2 \\
1
\end{array}\right) \lambda_{1}(x) & \cdots & 0 & 0 \\
\vdots & \vdots & \vdots & \ddots & \vdots & \vdots \\
\frac{(2 n-5) ! !}{2^{n-2}} & \lambda_{n-2}(x) & \left(\begin{array}{c}
n-2 \\
1
\end{array}\right) \lambda_{n-3}(x) & \cdots & \lambda_{0}(x) & 0 \\
\frac{(2 n-3) ! !}{2^{n-1}} & \lambda_{n-1}(x) & \left(\begin{array}{c}
n-1 \\
1
\end{array}\right) \lambda_{n-2}(x) & \cdots & \left(\begin{array}{c}
n-1 \\
n-2
\end{array}\right) \lambda_{1}(x) & \lambda_{0}(x) \\
\frac{(2 n-1) ! !}{2^{n}} & \lambda_{n}(x) & \left(\begin{array}{c}
n \\
1
\end{array}\right) \lambda_{n-1}(x) & \cdots & \left(\begin{array}{c}
n \\
n-2
\end{array}\right) \lambda_{2}(x) & \left(\begin{array}{c}
n \\
n-1
\end{array}\right) \lambda_{1}(x)
\end{array}\right),
$$

by the recursive relation

$$
\sum_{r=0}^{n}\left(\begin{array}{l}
n \\
r
\end{array}\right) \lambda_{n-r}(x) h_{r}(x)=-\frac{(2 n-1) ! !}{2^{n}}
$$

and by the explicit formula

$$
h_{n}(x)=n ! \sum_{k=0}^{n} \frac{[2(n-k)-1] ! !}{[2(n-k)] ! !} \frac{\lambda_{k}(-x)}{k !}
$$

for $n \geq 0$, where

$$
\lambda_{k}(x)=\frac{1}{2^{k}} \sum_{\ell=0}^{k}\left[\sum_{p=0}^{\ell}(-1)^{p}\left(\begin{array}{l}
\ell \\
p
\end{array}\right) \prod_{q=0}^{k-1}(p+2 q)\right] \frac{x^{\ell}}{\ell !}, \quad k \geq 0 .
$$

\section{LEMMAS}

In order to prove our main results, we recall several lemmas below.

Lemma 2.1 ([11, Theorem 1.4]). For $n \geq 0$, we have

$$
\frac{\mathrm{d}^{n}\left(e^{\sqrt{u}}\right)}{\mathrm{d} u^{n}}=e^{\sqrt{u}} \frac{(-1)^{n}}{(2 u)^{n}} \sum_{k=0}^{n}(-1)^{k}(2 n-2 k-1) ! !\left(\begin{array}{c}
2 n-k-1 \\
2(n-k)
\end{array}\right) u^{k / 2} .
$$

Lemma 2.2 ([14, p. 171, Theorem 12.1]). If $b_{\alpha}$ and $a_{k}$ are a collection of constants independent of $n$, then

$$
a_{n}=\sum_{\alpha=0}^{n} S(n, \alpha) b_{\alpha} \quad \text { if and only if } \quad b_{n}=\sum_{k=0}^{n} s(n, k) a_{k} .
$$

Lemma 2.3 ([14, p. 83, Eq. (7.12)]). If $a_{k}$ and $b_{k}$ for $k \geq 0$ are a collection of constants independent of $n$ such that $n \geq k \geq 0$, then

$$
a(n)=\sum_{k=0}^{n}(-1)^{k}\left(\begin{array}{l}
n \\
k
\end{array}\right) b(k) \quad \text { if and only if } b(n)=\sum_{k=0}^{n}(-1)^{k}\left(\begin{array}{l}
n \\
k
\end{array}\right) a(k) .
$$

Lemma 2.4 ([3, pp. 134 and 139]). For $n \geq k \geq 0$, the Bell polynomials of the second kind, or say, partial Bell polynomials, denoted by $\mathrm{B}_{n, k}\left(x_{1}, x_{2}, \ldots, x_{n-k+1}\right)$, are defined by

$$
\mathrm{B}_{n, k}\left(x_{1}, x_{2}, \ldots, x_{n-k+1}\right)=\sum_{\substack{1 \leq i \leq n, \ell_{i} \in\{0\} \cup \mathbb{N} \\ \sum_{i=1}^{n} i \ell_{i}=n \\ \sum_{i=1}^{n} \ell_{i}=k}} \frac{n !}{\prod_{i=1}^{n-k+1} \ell_{i} !} \prod_{i=1}^{n-k+1}\left(\frac{x_{i}}{i !}\right)^{\ell_{i}}
$$


The Fà̀ di Bruno formula can be described in terms of the Bell polynomials of the second kind $\mathrm{B}_{n, k}\left(x_{1}, x_{2}, \ldots, x_{n-k+1}\right)$ by

$$
\frac{\mathrm{d}^{n}}{\mathrm{~d} t^{n}} f \circ h(t)=\sum_{k=0}^{n} f^{(k)}(h(t)) \mathrm{B}_{n, k}\left(h^{\prime}(t), h^{\prime \prime}(t), \ldots, h^{(n-k+1)}(t)\right) .
$$

Lemma 2.5 ([3, p. 135]). For $n \geq k \geq 0$, we have

$$
\mathrm{B}_{n, k}\left(a b x_{1}, a b^{2} x_{2}, \ldots, a b^{n-k+1} x_{n-k+1}\right)=a^{k} b^{n} \mathrm{~B}_{n, k}\left(x_{1}, x_{2}, \ldots, x_{n-k+1}\right),
$$

where $a$ and $b$ are any complex numbers.

Lemma 2.6 ([9, Remark 1]). For $n \geq k \geq 0$, we have

$$
\begin{aligned}
\mathrm{B}_{n, k}\left(1,1-\lambda,(1-\lambda)(1-2 \lambda), \ldots, \prod_{\ell=0}^{n-k}(1-\ell \lambda)\right) \\
=\frac{(-1)^{k}}{k !} \sum_{\ell=0}^{k}(-1)^{\ell}\left(\begin{array}{c}
k \\
\ell
\end{array}\right) \prod_{q=0}^{n-1}(\ell-q \lambda) .
\end{aligned}
$$

Lemma 2.7 ([1, p. 40, Entry 5)]). Let $p=p(x)$ and $q=q(x) \neq 0$ be two differentiable functions. Then

$$
\left[\frac{p(x)}{q(x)}\right]^{(k)}=\frac{(-1)^{k}}{q^{k+1}}\left|\begin{array}{cccccc}
p & q & 0 & \cdots & 0 & 0 \\
p^{\prime} & q^{\prime} & q & \cdots & 0 & 0 \\
p^{\prime \prime} & q^{\prime \prime} & \left(\begin{array}{c}
2 \\
1
\end{array}\right) q^{\prime} & \cdots & 0 & 0 \\
\vdots & \vdots & \vdots & \ddots & \vdots & \vdots \\
p^{(k-2)} & q^{(k-2)} & \left(\begin{array}{c}
k-2 \\
1
\end{array}\right) q^{(k-3)} & \ldots & q & 0 \\
p^{(k-1)} & q^{(k-1)} & \left(\begin{array}{c}
k-1 \\
1
\end{array}\right) q^{(k-2)} & \ldots & \left(\begin{array}{c}
k-1 \\
k-2
\end{array}\right) q^{\prime} & q \\
p^{(k)} & q^{(k)} & \left(\begin{array}{c}
k \\
1
\end{array}\right) q^{(k-1)} & \ldots & \left(\begin{array}{c}
k \\
k-2
\end{array}\right) q^{\prime \prime} & \left(\begin{array}{c}
k \\
k-1
\end{array}\right) q^{\prime}
\end{array}\right|
$$

for $k \geq 0$. In other words, the formula 2.4 can be rewritten as

$$
\frac{\mathrm{d}^{k}}{\mathrm{~d} x^{k}}\left[\frac{p(x)}{q(x)}\right]=\frac{(-1)^{k}}{q^{k+1}(x)}\left|W_{(k+1) \times(k+1)}(x)\right|,
$$

where $\left|W_{(k+1) \times(k+1)}(x)\right|$ denotes the determinant of the $(k+1) \times(k+1)$ matrix

$$
W_{(k+1) \times(k+1)}(x)=\left(U_{(k+1) \times 1}(x) \quad V_{(k+1) \times k}(x)\right),
$$

the quantity $U_{(k+1) \times 1}(x)$ is a $(k+1) \times 1$ matrix whose elements $u_{\ell, 1}(x)=p^{(\ell-1)}(x)$ for $1 \leq \ell \leq k+1$, and $V_{(k+1) \times k}(x)$ is a $(k+1) \times k$ matrix whose elements

$$
v_{i, j}(x)= \begin{cases}\left(\begin{array}{l}
i-1 \\
j-1
\end{array}\right) q^{(i-j)}(x), & i-j \geq 0 \\
0, & i-j<0\end{cases}
$$

for $1 \leq i \leq k+1$ and $1 \leq j \leq k$. 
Lemma 2.8 ([2, p. 222, Theorem] and [12, Remark 3]). Let $M_{0}=1$ and

$$
M_{n}=\left|\begin{array}{cccccc}
m_{1,1} & m_{1,2} & 0 & \cdots & 0 & 0 \\
m_{2,1} & m_{2,2} & m_{2,3} & \cdots & 0 & 0 \\
m_{3,1} & m_{3,2} & m_{3,3} & \cdots & 0 & 0 \\
\vdots & \vdots & \vdots & \ddots & \vdots & \vdots \\
m_{n-2,1} & m_{n-2,2} & m_{n-2,3} & \cdots & m_{n-2, n-1} & 0 \\
m_{n-1,1} & m_{n-1,2} & m_{n-1,3} & \cdots & m_{n-1, n-1} & m_{n-1, n} \\
m_{n, 1} & m_{n, 2} & m_{n, 3} & \cdots & m_{n, n-1} & m_{n, n}
\end{array}\right|
$$

for $n \in \mathbb{N}$. Then the sequence $M_{n}$ for $n \geq 0$ satisfies $M_{1}=m_{1,1}$ and

$$
M_{n}=m_{n, n} M_{n-1}+\sum_{r=1}^{n-1}(-1)^{n-r} m_{n, r}\left(\prod_{j=r}^{n-1} m_{j, j+1}\right) M_{r-1}, \quad n \geq 2 .
$$

\section{Proofs of MAin Results}

Now we are in a position to prove our main results.

Proof of Theorem 1.4. By virtue of Lemma 2.1, the formula 1.2) implies that

$$
\begin{aligned}
h_{n}(x)= & \frac{1}{n !} \frac{x}{e^{x}}\left(\frac{\mathrm{d}}{\mathrm{d} u}\right)^{n}\left(u^{n-1 / 2} e^{\sqrt{u}}\right), \quad u=x^{2} \\
= & \frac{1}{n !} \frac{x}{e^{x}} \sum_{k=0}^{n}\left(\begin{array}{l}
n \\
k
\end{array}\right)\left(u^{n-1 / 2}\right)^{(n-k)}\left(e^{\sqrt{u}}\right)^{(k)} \\
= & \frac{1}{n !} \frac{x}{e^{x}} \sum_{k=0}^{n}\left(\begin{array}{l}
n \\
k
\end{array}\right)\left\langle n-\frac{1}{2}\right\rangle_{n-k} u^{k-1 / 2} \frac{(-1)^{k}}{(2 u)^{k}} \\
& \times e^{\sqrt{u}} \sum_{\ell=0}^{k}(-1)^{\ell}[2(k-\ell)-1] ! !\left(\begin{array}{c}
2 k-\ell-1 \\
2(k-\ell)
\end{array}\right) u^{\ell / 2} \\
= & \frac{1}{n !} \sum_{k=0}^{n} \frac{(-1)^{k}}{2^{k}}\left(\begin{array}{l}
n \\
k
\end{array}\right)\left\langle n-\frac{1}{2}\right\rangle_{n-k} \sum_{\ell=0}^{k}(-1)^{\ell}[2(k-\ell)-1] ! !\left(\begin{array}{c}
2 k-\ell-1 \\
2(k-\ell)
\end{array}\right) x^{\ell} .
\end{aligned}
$$

The proof of Theorem 1.4 is complete.

Proof of Theorem 1.5. The identity (1.6) in Theorem 1.3 can be rearranged as

$$
-\frac{1}{2^{n}} \sum_{k=0}^{n}\left(\begin{array}{l}
n \\
k
\end{array}\right) 2^{k}(2 n-2 k-3) ! ! h_{k}(x)=\sum_{k=0}^{n} s(n, k) \frac{B_{k}(x)}{2^{k}} .
$$

Further utilizing Lemma 2.2 yields

$$
\frac{B_{n}(x)}{2^{n}}=-\sum_{k=0}^{n} S(n, k) \frac{1}{2^{k}} \sum_{\ell=0}^{k}\left(\begin{array}{l}
k \\
\ell
\end{array}\right) 2^{\ell}(2 k-2 \ell-3) ! ! h_{\ell}(x) .
$$

The proof of Theorem 1.5 is complete.

Proof of Theorem 1.6. Interchanging the order of sums in the right hand side of the identity 1.4 in Theorem 1.2 arrives at

$$
(-1)^{n} B_{n}(x)=\sum_{k=0}^{n}(-1)^{k}\left(\begin{array}{l}
n \\
k
\end{array}\right)(-2)^{k} \sum_{\ell=0}^{k} S(k, \ell)(-1)^{\ell} h_{\ell}(x) .
$$


Further applying Lemma 2.3 leads to

$$
(-2)^{n} \sum_{\ell=0}^{n} S(n, \ell)(-1)^{\ell} h_{\ell}(x)=\sum_{k=0}^{n}(-1)^{k}\left(\begin{array}{l}
n \\
k
\end{array}\right)(-1)^{k} B_{k}(x)=\sum_{k=0}^{n}\left(\begin{array}{l}
n \\
k
\end{array}\right) B_{k}(x)
$$

which is equivalent to 1.3 .

Employing Lemma 2.2 in 1.3 produces

$$
(-1)^{n} h_{n}(x)=\sum_{k=0}^{n} s(n, k) \frac{(-1)^{k}}{2^{k}} \sum_{\ell=0}^{k}\left(\begin{array}{l}
k \\
\ell
\end{array}\right) B_{\ell}(x) .
$$

The proof of Theorem 1.6 is complete.

Proof of Theorem 1.7. By straightforward computation, we obtain

$$
\begin{aligned}
& \frac{\mathrm{d}^{k}}{\mathrm{~d} t^{k}}\left[(1-t)^{-1 / 2}\right]=\left\langle-\frac{1}{2}\right\rangle_{k}(-1)^{k}(1-t)^{-1 / 2-k} \\
& \rightarrow\left\langle-\frac{1}{2}\right\rangle_{k}(-1)^{k}=\left(\frac{1}{2}\right)_{k}=\frac{(2 k-1) ! !}{2^{k}}, \quad t \rightarrow 0
\end{aligned}
$$

and, when denoting $u=u(t)=(1-t)^{-1 / 2}$ and making use of the formulas 2.1 and 2.2,

$$
\begin{gathered}
\frac{\mathrm{d}^{k} \exp \left[-x(1-t)^{-1 / 2}\right]}{\mathrm{d} t^{k}}=\sum_{\ell=0}^{k}\left(e^{-x u}\right)^{(\ell)} \mathrm{B}_{k, \ell}\left(u^{\prime}(t), u^{\prime \prime}(t), \ldots, u^{(k-\ell+1)}(t)\right) \\
=\sum_{\ell=0}^{k}(-x)^{\ell} e^{-x u} \mathrm{~B}_{k, \ell}\left(\left\langle-\frac{1}{2}\right\rangle_{1} \frac{-1}{(1-t)^{3 / 2}},\left\langle-\frac{1}{2}\right\rangle_{2} \frac{1}{(1-t)^{5 / 2}}, \ldots,\right. \\
\left.\quad\left\langle-\frac{1}{2}\right\rangle_{k-\ell+1} \frac{(-1)^{k-\ell+1}}{(1-t)^{k-\ell+3 / 2}}\right) \\
\rightarrow e^{-x} \sum_{\ell=0}^{k}(-x)^{\ell} \mathrm{B}_{k, \ell}\left(\frac{1 ! !}{2}, \frac{3 ! !}{2^{2}}, \ldots, \frac{(2 k-2 \ell+1) ! !}{2^{k-\ell+1}}\right), \quad t \rightarrow 0 \\
=\frac{e^{-x}}{2^{k}} \sum_{\ell=0}^{k}(-x)^{\ell} \mathrm{B}_{k, \ell}(1 ! !, 3 ! !, \ldots,(2 k-2 \ell+1) ! !) .
\end{gathered}
$$

Taking $\lambda=-2$ in 2.3 gives

$$
\mathrm{B}_{n, k}(1 ! !, 3 ! !, \ldots,(2 n-2 k+1) ! !)=\frac{(-1)^{k}}{k !} \sum_{\ell=0}^{k}(-1)^{\ell}\left(\begin{array}{c}
k \\
\ell
\end{array}\right) \prod_{q=0}^{n-1}(\ell+2 q)
$$

for $n \geq k \geq 0$. Therefore, we obtain

$$
\lim _{t \rightarrow 0} \frac{\mathrm{d}^{k} \exp \left[-x(1-t)^{-1 / 2}\right]}{\mathrm{d} t^{k}}=\frac{e^{-x}}{2^{k}} \sum_{\ell=0}^{k} \frac{x^{\ell}}{\ell !} \sum_{p=0}^{\ell}(-1)^{p}\left(\begin{array}{l}
\ell \\
p
\end{array}\right) \prod_{q=0}^{k-1}(p+2 q)=e^{-x} \lambda_{k}(x) .
$$

The equation 1.1 means that

$$
h_{n}(x)=\lim _{t \rightarrow 0} \frac{\mathrm{d}^{n}}{\mathrm{~d} t^{n}} \frac{(1-t)^{-1 / 2}}{\exp \left[x\left(1-(1-t)^{-1 / 2}\right)\right]}=e^{-x} \lim _{t \rightarrow 0} \frac{\mathrm{d}^{n}}{\mathrm{~d} t^{n}} \frac{(1-t)^{-1 / 2}}{\exp \left[-x(1-t)^{-1 / 2}\right]} .
$$


Further applying the formula 2.5 to

$$
p(t)=(1-t)^{-1 / 2} \text { and } q(t)=\exp \left[-x(1-t)^{-1 / 2}\right]
$$

results in

$$
\begin{aligned}
& h_{n}(x)=(-1)^{n} e^{-x} \lim _{t \rightarrow 0} \exp \left[(n+1) x(1-t)^{-1 / 2}\right] \\
& \times \lim _{t \rightarrow 0}\left|\begin{array}{cccccc}
p & q & 0 & \cdots & 0 & 0 \\
p^{\prime} & q^{\prime} & q & \cdots & 0 & 0 \\
p^{\prime \prime} & q^{\prime \prime} & \left(\begin{array}{l}
2 \\
1
\end{array}\right) q^{\prime} & \cdots & 0 & 0 \\
\vdots & \vdots & \vdots & \ddots & \vdots & \vdots \\
p^{(n-2)} & q^{(n-2)} & \left(\begin{array}{c}
n-2 \\
1
\end{array}\right) q^{(n-3)} & \cdots & q & 0 \\
p^{(n-1)} & q^{(n-1)} & \left(\begin{array}{c}
n-1 \\
1
\end{array}\right) q^{(n-2)} & \cdots & \left(\begin{array}{c}
n-1 \\
n-2
\end{array}\right) q^{\prime} & q \\
p^{(n)} & q^{(n)} & \left(\begin{array}{c}
n \\
1
\end{array}\right) q^{(n-1)} & \cdots & \left(\begin{array}{c}
n \\
n-2
\end{array}\right) q^{\prime \prime} & \left(\begin{array}{c}
n \\
n-1
\end{array}\right) q^{\prime}
\end{array}\right| \\
& =(-1)^{n} e^{n x}\left|\begin{array}{cccccc}
1 & q_{0}(x) & 0 & \cdots & 0 & 0 \\
\frac{1 ! !}{2} & q_{1}(x) & q_{0}(x) & \cdots & 0 & 0 \\
\frac{3 ! !}{2^{2}} & q_{2}(x) & \left(\begin{array}{l}
2 \\
1
\end{array}\right) q_{1}(x) & \cdots & 0 & 0 \\
\vdots & \vdots & \vdots & \ddots & \vdots & \vdots \\
\frac{(2 n-5) ! !}{2^{2 n-2}} & q_{n-2}(x) & \left(\begin{array}{c}
n-2 \\
1
\end{array}\right) q_{n-3}(x) & \cdots & q_{0}(x) & 0 \\
\frac{(2 n-3) ! !}{2^{2 n-1}} & q_{n-1}(x) & \left(\begin{array}{c}
n-1 \\
1
\end{array}\right) q_{n-2}(x) & \cdots & \left(\begin{array}{c}
n-1 \\
n-2
\end{array}\right) q_{1}(x) & q_{0}(x) \\
\frac{(2 n-1) ! !}{2^{n}} & q_{n}(x) & \left(\begin{array}{c}
n \\
1
\end{array}\right) q_{n-1}(x) & \cdots & \left(\begin{array}{c}
n \\
n-2
\end{array}\right) q_{2}(x) & \left(\begin{array}{c}
n \\
n-1
\end{array}\right) q_{1}(x)
\end{array}\right| \\
& =(-1)^{n}\left|\begin{array}{cccccc}
1 & \lambda_{0}(x) & 0 & \cdots & 0 & 0 \\
\frac{1 ! !}{2} & \lambda_{1}(x) & \lambda_{0}(x) & \cdots & 0 & 0 \\
\frac{3 ! !}{2^{2}} & \lambda_{2}(x) & \left(\begin{array}{l}
2 \\
1
\end{array}\right) \lambda_{1}(x) & \cdots & 0 & 0 \\
\vdots & \vdots & \vdots & \ddots & \vdots & \vdots \\
\frac{(2 n-5) ! !}{2^{n-2}} & \lambda_{n-2}(x) & \left(\begin{array}{c}
n-2 \\
1
\end{array}\right) \lambda_{n-3}(x) & \cdots & \lambda_{0}(x) & 0 \\
\frac{(2 n-3) ! !}{2^{n-1}} & \lambda_{n-1}(x) & \left(\begin{array}{c}
n-1 \\
1
\end{array}\right) \lambda_{n-2}(x) & \cdots & \left(\begin{array}{c}
n-1 \\
n-2
\end{array}\right) \lambda_{1}(x) & \lambda_{0}(x) \\
\frac{(2 n-1) ! !}{2^{n}} & \lambda_{n}(x) & \left(\begin{array}{c}
n \\
1
\end{array}\right) \lambda_{n-1}(x) & \cdots & \left(\begin{array}{c}
n \\
n-2
\end{array}\right) \lambda_{2}(x) & \left(\begin{array}{c}
n \\
n-1
\end{array}\right) \lambda_{1}(x)
\end{array}\right|,
\end{aligned}
$$

where $q_{k}(x)=e^{-x} \lambda_{k}(x)$ for $k \geq 0$. The determinantal expression 1.10$)$ is proved.

Applying 2.6. to the determinantal expression (1.10) and considering $\lambda_{0}(x)=1$ derive

$$
\begin{aligned}
(-1)^{n} h_{n}(x)=n \lambda_{1}(x)(-1)^{n-1} h_{n-1}(x)+(-1)^{n-1} \frac{(2 n-1) ! !}{2^{n}} & \\
& +\sum_{r=2}^{n}(-1)^{n-r+1}\left(\begin{array}{c}
n \\
r-2
\end{array}\right) \lambda_{n-r+2}(x)(-1)^{r-2} h_{r-2}(x), \quad n \geq 2 .
\end{aligned}
$$

This can be reformulated as 1.11).

From the equation (1.1) and the above arguments, it follows that

$$
\begin{gathered}
h_{n}(x)=e^{-x} \lim _{t \rightarrow 0} \frac{\mathrm{d}^{n}}{\mathrm{~d} t^{n}}\left[\frac{1}{\sqrt{1-t}} \exp \left(\frac{x}{\sqrt{1-t}}\right)\right] \\
=e^{-x} \lim _{t \rightarrow 0} \sum_{k=0}^{n}\left(\begin{array}{l}
n \\
k
\end{array}\right) \frac{\mathrm{d}^{n-k}}{\mathrm{~d} t^{n-k}} \frac{1}{\sqrt{1-t}} \frac{\mathrm{d}^{k}}{\mathrm{~d} t^{k}} \exp \left(\frac{x}{\sqrt{1-t}}\right)
\end{gathered}
$$




$$
\begin{gathered}
=e^{-x} \sum_{k=0}^{n}\left(\begin{array}{l}
n \\
k
\end{array}\right) \lim _{t \rightarrow 0} \frac{\mathrm{d}^{n-k}}{\mathrm{~d} t^{n-k}} \frac{1}{\sqrt{1-t}} \lim _{t \rightarrow 0} \frac{\mathrm{d}^{k}}{\mathrm{~d} t^{k}} \exp \left(\frac{x}{\sqrt{1-t}}\right) \\
=e^{-x} \sum_{k=0}^{n}\left(\begin{array}{l}
n \\
k
\end{array}\right) \frac{(2 n-2 k-1) ! !}{2^{n-k}} \frac{e^{x}}{2^{k}} \sum_{\ell=0}^{k} \frac{(-x)^{\ell}}{\ell !} \sum_{p=0}^{\ell}(-1)^{p}\left(\begin{array}{l}
\ell \\
p
\end{array}\right) \prod_{q=0}^{k-1}(p+2 q) \\
=\frac{1}{2^{n}} \sum_{k=0}^{n}\left(\begin{array}{l}
n \\
k
\end{array}\right)(2 n-2 k-1) ! ! \sum_{\ell=0}^{k} \frac{(-1)^{\ell}}{\ell !} x^{\ell} \sum_{p=0}^{\ell}(-1)^{p}\left(\begin{array}{l}
\ell \\
p
\end{array}\right) \prod_{q=0}^{k-1}(p+2 q)
\end{gathered}
$$

which can be rewritten as 1.12 . The proof of Theorem 1.7 is complete.

\section{Remarks}

Finally we list several remarks about our main results and other things.

Remark 4.1. Recently a new inversion theorem was discovered in [13, Theorem 4.3] which can be reformulated as that

$$
s_{n}=\sum_{k=1}^{n}\left(\begin{array}{c}
k \\
n-k
\end{array}\right) S_{k} \quad \text { if and only if } \quad(-1)^{n} n S_{n}=\sum_{k=1}^{n}(-1)^{k} k\left(\begin{array}{c}
2 n-k-1 \\
n-1
\end{array}\right) s_{k},
$$

where $s_{k}$ and $S_{k}$ are two sequences independent of $n$ such that $n \geq k \geq 1$.

Remark 4.2. The identity 1.4 is slightly different from 1.8 .

Remark 4.3. The identity 1.9 is obviously simpler than 1.5 in their forms.

Remark 4.4. Comparing (1.7) with 1.11) reveals that

$$
\sum_{\ell=0}^{n-k} \frac{s(n-k, \ell)}{2^{\ell}} B_{\ell}(-x)=-\lambda_{n-k}(x)
$$

that is,

$$
\sum_{\ell=0}^{n} s(n, \ell) \frac{B_{\ell}(x)}{2^{\ell}}=-\lambda_{n}(-x)
$$

Further considering Lemma 2.2 deduces

$$
B_{n}(x)=-2^{n} \sum_{\ell=0}^{n} S(n, \ell) \lambda_{\ell}(-x) .
$$

\section{REFERENCES}

[1] N. Bourbaki, Functions of a Real Variable, Elementary Theory, Translated from the 1976 French original by Philip Spain. Elements of Mathematics (Berlin). Springer-Verlag, Berlin, 2004; Available online at http://dx.doi.org/10.1007/978-3-642-59315-4

[2] N. D. Cahill, J. R. D'Errico, D. A. Narayan, and J. Y. Narayan, Fibonacci determinants, College Math. J. 3 (2002), 221-225; Available online at http://dx.doi.org/10.2307/1559033

[3] L. Comtet, Advanced Combinatorics: The Art of Finite and Infinite Expansions, Revised and Enlarged Edition, D. Reidel Publishing Co., 1974.

[4] A. Erdélyi, W. Magnus, F. Oberhettinger, and F. G. Tricomi, Higher Transcendental Functions, Vol. III. Based on notes left by Harry Bateman. Reprint of the 1955 original. Robert E. Krieger Publishing Co., Inc., Melbourne, Fla., 1981.

[5] T. Kim, D. V. Dolgy, D. S. Kim, H. I. Kwon, and J. J. Seo, Differential equations arising from certain Sheffer sequence, J. Comput. Anal. Appl. 23 (2017), no. 8, 1359-1367.

[6] W.-X. Ma, Bilinear equations, Bell polynomials and linear superposition principle, J. Phys. Conf. Ser. 411 (2013), no. 1, Aricle ID 012021, 11 pages; Available online at http://dx.doi. org/10.1088/1742-6596/411/1/012021. 
[7] W.-X. Ma, Bilinear equations and resonant solutions characterized by Bell polynomials, Rep. Math. Phys. 72 (2013), no. 1, 41-56; Available online at https://doi.org/10.1016/ S0034-4877(14)60003-3

[8] W.-X. Ma, Trilinear equations, Bell polynomials, and resonant solutions, Front. Math. China 8 (2013), no. 5, 1139-1156; Available online at https://doi.org/10.1007/ s11464-013-0319-5

[9] F. Qi and B.-N. Guo, Viewing some ordinary differential equations from the angle of derivative polynomials, Preprints 2016, 2016100043, 12 pages; Available online at http: //dx.doi.org/10.20944/preprints201610.0043.v1

[10] F. Qi, D. Lim, and B.-N. Guo, Explicit formulas and identities for the Bell polynomials and a sequence of polynomials applied to differential equations, Rev. R. Acad. Cienc. Exactas Fís. Nat. Ser. A Mat. RACSAM (2018), in press; Available online at http://dx.doi.org/10. 1007/s13398-017-0427-2

[11] F. Qi, X.-T. Shi, F.-F. Liu, and D. V. Kruchinin, Several formulas for special values of the Bell polynomials of the second kind and applications, J. Appl. Anal. Comput. 7 (2017), no. 3, 857-871; Available online at http://dx.doi.org/10.11948/2017054

[12] F. Qi, J.-L. Zhao, and B.-N. Guo, Closed forms for derangement numbers in terms of the Hessenberg determinants, Rev. R. Acad. Cienc. Exactas Fís. Nat. Ser. A Mat. RACSAM 112 (2018), in press; Available online at http://dx.doi.org/10.1007/s13398-017-0401-z

[13] F. Qi, Q. Zou, and B.-N. Guo, Some identities and a matrix inverse related to the Chebyshev polynomials of the second kind and the Catalan numbers, Preprints 2017, 2017030209, 25 pages; Available online at http://dx.doi.org/10.20944/preprints201703.0209.v2

[14] J. Quaintance and H. W. Gould, Combinatorial Identities for Stirling Numbers. The unpublished notes of H. W. Gould. With a foreword by George E. Andrews. World Scientific Publishing Co. Pte. Ltd., Singapore, 2016. 